2011-05-01

\title{
Right hemisphere dominance in visual statistical learning
}

Roser, ME::0000-0001-6322-9863

http://hdl.handle.net/10026.1/14638

10.1162/jocn.2010.21508

Journal of Cognitive Neuroscience

MIT Press - Journals

All content in PEARL is protected by copyright law. Author manuscripts are made available in accordance with publisher policies. Please cite only the published version using the details provided on the item record or document. In the absence of an open licence (e.g. Creative Commons), permissions for further reuse of content should be sought from the publisher or author. 


\title{
Right Hemisphere Dominance in Visual Statistical Learning
}

\author{
Matthew E. Roser ${ }^{1}$, József Fiser ${ }^{2}$, Richard N. Aslin ${ }^{3}$, \\ and Michael S. Gazzaniga ${ }^{4}$
}

\begin{abstract}
Several studies report a right hemisphere advantage for visuospatial integration and a left hemisphere advantage for inferring conceptual knowledge from patterns of covariation. The present study examined hemispheric asymmetry in the implicit learning of new visual feature combinations. A split-brain patient and normal control participants viewed multishape scenes presented in either the right or the left visual fields. Unbeknownst to the participants, the scenes were composed from a random combination of fixed pairs of shapes. Subsequent testing found that
\end{abstract}

\section{INTRODUCTION}

The human visual system must make sense of enormous amounts of information contained in the visual array. This includes spatial and temporal information about objects, their movements, and their associations with other objects in cluttered visual scenes. To enable the recognition of objects in new environments, the visual system must be able to extract invariant visual features (Biederman, 1987; Marr, 1982). Although some features can be extracted automatically by low-level visual analyzers, recognizing many visual features requires a learning mechanism that links two or more elements in the array into meaningful chunks. Indeed, a growing body of research suggests that encoding associations between stimuli can occur through unsupervised observational learning without any specific feedback about the configuration or number of element combinations. This process has been shown with speech streams in adults (Saffran, Newport, \& Aslin, 1996), infants (Saffran, Aslin, \& Newport, 1996), and tamarins (Hauser, Newport, \& Aslin, 2001); in visually presented objects in adults (Fiser, Scholl, \& Aslin, 2007; Fiser \& Aslin, 2001, 2002a, 2005) and infants (Fiser \& Aslin, 2002b; Kirkham, Slemmer, \& Johnson, 2002); and in other modalities (Conway \& Christiansen, 2005). Together, these findings of sensitivity to co-occurrence statistics between elements are referred to as examples of statistical learning (Perruchet \& Pacton, 2006).

Studies of statistical learning in the visual modality have presented stimuli in two fundamental ways. Focusing on

${ }^{1}$ University of Plymouth, UK, ${ }^{2}$ Brandeis University, ${ }^{3}$ University of Rochester, ${ }^{4}$ University of California, Santa Barbara control participants could discriminate fixed-pair shapes from randomly combined shapes when presented in either visual field. The split-brain patient performed at chance except when both the practice and the test displays were presented in the left visual field (right hemisphere). These results suggest that the statistical learning of new visual features is dominated by visuospatial processing in the right hemisphere and provide a prediction about how fMRI activation patterns might change during unsupervised statistical learning.

the ability to learn temporal statistics, Fiser and Aslin (2002a) and Kirkham et al. (2002) presented participants with sequences of shapes and found that observers were sensitive to the temporal-order statistics of the sequences, such as the joint probabilities of successive shape pairs. Focusing on the spatial statistics, Fiser and Aslin (2001, 2002b, 2005) presented participants with a family of scenes, each of which contained multiple shapes arranged in a grid. In each scene, the spatial arrangement of the entire array of shapes was changed, but the spatial arrangement between particular pairs of shapes (base pairs) was always preserved. After passive observation of these statistically constrained arrays of shapes, observers could distinguish base pairs from non-base pairs, demonstrating learning of spatial relations among the shapes that were used to compose the scenes.

The finding that statistical learning of shape-pair associations occurs when participants are given no particular task instructions other than to observe the stimuli has been taken to suggest that these implicit-learning paradigms explore a basic mechanism of human learning that occurs with little explicit top-down input from higher cognitive processes (Fiser \& Aslin, 2005). Once such bottom-up associations are learned, however, they become available as implicit top-down information that can influence the outcome of subsequent learning by constraining the statistics that are computed. This implicit top-down effect can lead to such things as visual chunk learning (Orban, Fiser, Aslin, \& Lengyel, 2008), a variety of mid-level perceptual effects, or even to emergence of explicit rules that guide other top-down processes (MacKenzie \& Fiser, 2008; Saffran, Pollak, Seibel, \& Shkolnik, 2007). 
Another view is that a variety of constraints, some of which trigger obligatory parsing of elements, bias the statistical learning process. For example, Creel, Newport, and Aslin (2004) found that regularities among temporally nonadjacent tones could be acquired if the tones were perceptually similar (in pitch and timbre), whereas statistics were not computed across elements that were temporally adjacent but in different perceptual groups. In the visual domain, Fiser et al. (2007) presented participants with displays in which the spatio-temporal properties of moving elements were manipulated so that participants perceived either streaming (continuous motion) of two pairs of distinctive objects behind a larger occluding object or the bouncing of two objects behind the occluding object. They found that the induced perception (streaming or bouncing) affected the statistical learning of associations between shapes, suggesting that statistical learning is constrained at the level at which representations of objects as spatiotemporal entities are formed. Other researchers have found that connectedness, a cue to perceptual grouping, modulates statistical learning of shape associations (Baker, Olson, \& Behrmann, 2004) and that overt attention to a subset of shapes (cued by color) can limit the domain over which statistical learning takes place (Turk-Browne, Junge, \& Scholl, 2005). Thus, both explicit and implicit mechanisms can play a role in statistical learning, even when there is no task other than passive observation and no feedback about the specific relations or number of elements that cohere statistically (see Perruchet \& Pacton, 2006).

The foregoing review is consistent with a process of statistical learning that begins with raw sensory input, continuously constrained by mid-level perceptual and attentional mechanisms, and then forms internal representations that themselves become constraints and can be used to interpret novel inputs. This learning process may or may not involve conscious awareness of the underlying statistical structures that have been learned, but the representation it forms becomes gradually more abstract so that it can subserve simple co-occurrence based associations as well as sophisticated chunking and rule learning. A key question, then, is what neural mechanism supports this type of statistical learning?

A large body of data from callosotomy (split-brain) patients suggest that the right hemisphere has an advantage over the left hemisphere in tasks that require visuospatial integration or discrimination (Corballis, 2003; Corballis, Funnell, \& Gazzaniga, 2002). This superiority is apparent in tests of mental rotation (Corballis \& Sergent, 1988), perception of illusory amodally completed contours (Corballis, Fendrich, Shapley, \& Gazzaniga, 1999), and block construction (Bogen \& Gazzaniga, 1965). Furthermore, the right hemisphere in split-brain patients has been shown previously to be critically involved in the extraction of structure from the interaction of visual elements. Roser, Fugelsang, Dunbar, Corballis, and Gazzaniga (2005) found that the right hemispheres of two callosotomy patients were sensitive to the spatio-temporal properties of interactions between objects crucial to the perception of causality in collision events. Conversely, the patient's left hemispheres were insensitive to manipulations of visuospatial properties but performed well on a task requiring simple explicit reasoning that the patient's right hemispheres failed. Thus, the right hemisphere sensitivity to spatial and temporal properties was observed in the absence of the ability to make inferences at the 2-year-old level, and basic perceptual and inferential components of understanding physical causality were doubly dissociated. Moreover, righthemispheric parietal and frontal areas were found to be differentially active for object interactions with differing spatio-temporal properties in a functional MRI study with neurologically normal participants (Fugelsang, Roser, Corballis, Gazzaniga, \& Dunbar, 2005), and a recent fMRI investigation of statistical learning reported activations predominantly in right hemisphere regions at an early stage of familiarization with visually presented sequences of shapes (Turk-Browne, Scholl, Chun, \& Johnson, 2009). Therefore, right-hemispheric processing is a good candidate for performing learning through observation of new visual features defined by spatial arrangement.

There is, however, evidence that supports the involvement of the left hemisphere, too, in this learning process. The isolated left hemisphere has a propensity for interpretation of complex stimuli and actions (Gazzaniga, 2000). In the split brain, the left hemisphere often generates explanations for events and categorizes stimuli, despite this strategy sometimes leading to suboptimal performance. For example, when each hemisphere in a split-brain participant was asked to predict the likely location of a forthcoming stimulus, the left hemisphere attempted to detect patterns in a random serial presentation and performed suboptimally (Wolford, Miller, \& Gazzaniga, 2000). The right hemisphere repeatedly chose the location at which stimuli appeared most often, thus maximizing performance. In other studies, left-hemispheric memory performance was also adversely affected by a tendency to falsely recognize novel pictures of scenes that were conceptually consistent with scenes presented for encoding (Phelps \& Gazzaniga, 1992). This proclivity of the left hemisphere for the generation of hypotheses about structure and meaning in the input it receives includes the actions of the isolated right hemisphere. When different scenes were presented to each hemisphere of split-brain patients and each hemisphere was asked to manually indicate an item consistent with that scene, the left hemisphere's explanation for the choice made by the right hemisphere was a confabulation upon the information contained in the scene presented to the left hemisphere (Gazzaniga, 2000). Thus, the left hemisphere generates hypotheses about patterns in the environment, elaborates on information it receives, and creates explanations for events that include the actions of an isolated right hemisphere. These high-level interpretations of visual input might well be necessary for learning new visual representations. 
The predominant role of the left hemisphere in tasks that involve conceptual knowledge and of the right hemisphere in tasks involving perceptual grouping raises two possibilities. First, statistical learning of the covariance of elements in visual scenes may be focused in regions of the right hemisphere, especially because statistical learning is often an implicit task with no conscious awareness of the underlying structures that have been extracted. Second, because the outcome generalizes to new scenes, statistical learning could involve both bottom-up right hemisphere learning and top-down left hemisphere recognition or even a strong dominance by the hypothesis-making left hemisphere.

To assess these two possibilities, we presented information about the covariance of visual elements separately to each of the cerebral hemispheres of a callosotomy (splitbrain) patient, known in the literature as V.P., and a group of callosally intact control participants. As cortical registration of visual input from each hemifield is lateralized to the contralateral hemisphere and only the anterior commissure and subcortical connections remain intact, each hemisphere in the split brain can be tested in relative isolation. Testing the divided brain can also allow otherwise indivisible components of a process to be dissociated. For instance, Baynes, Eliassen, Lutsep, and Gazzaniga (1998) found that spoken and written language output can be controlled independently by the two disconnected hemispheres. The double dissociation between explicit causal reasoning and causal perception found by Roser et al. (2005) suggests that separate mechanisms that depend critically on opposite hemispheres of the brain mediate these two processes, and a similar dissociation may be revealed in the present study of visual feature learning. Importantly, if it is found that the isolated right hemisphere performs well on a statistical learning task but that the left hemisphere does not, it follows that processes supported by the right hemisphere are both necessary and sufficient for statistical learning of new visual features. Put simply, the statistical learning of covariance between visual elements in the divided brain may depend on right-hemispheric perceptual processes and may proceed in the absence of high-level conceptual knowledge supported by the left hemisphere. These effects would manifest as differences in split-brain patient performance for visual input displayed in each visual hemifield. Intact callosal transmission in the control participants should ensure that visual information presented to either of the visual hemifields is available to visuospatial processes supported primarily by the right hemisphere. As this pathway is available in control participants, we expected no effect of manipulation of the visual field of presentation for this group.

\section{EXPERIMENT 1}

To make valid inferences about hemispheric involvement in statistical learning on the basis of performance with stim- uli lateralized to a single hemisphere in the split brain, it was first necessary to determine that above-chance performance in normal participants was possible with stimuli presented in the retinal periphery. All prior studies of visual statistical learning were conducted with free viewing, thereby presenting the entire set of input scenes to both hemispheres.

\section{Methods}

\section{Participants}

Sixteen undergraduate students (mean age approximately 20 years) participated in the experiment for course credit. All participants had normal or corrected-to-normal vision. Informed consent to participate was obtained, and all studies were approved by the Dartmouth and University of Plymouth ethics committees and have therefore been performed in accordance with the ethical standards laid down in the 1964 Declaration of Helsinki.

\section{Stimuli}

The stimuli used were the same as those used by Fiser and Aslin (2002b). Twelve complex two-dimensional shapes were created. Shapes were black on a white background and were displayed within a $3 \times 3$ grid using PsyScope software (Cohen, MacWhinney, Flatt, \& Provost, 1993). The grids were displayed either to the left or to the right of a central fixation point and were thus lateralized to one visual hemifield on each trial. The edge of the grid nearest to the fovea was located approximately $2^{\circ}$ into the peripheral visual field. The grid subtended $12^{\circ}$ of visual angle, and the entire display was viewed from $57 \mathrm{~cm}$.

\section{Procedure}

The experiment consisted of familiarization and test phases. Unbeknownst to the participants, the multishape scenes were randomly composed from six base pairs, each pair comprising two of the shapes in an invariant spatial relationship. Figure 1 shows three base pairs (horizontal, oblique, and vertical) combined to form one multishape scene.

These six base pairs were then further divided into two sets of three pairs, and the scenes for the familiarization phase were created by configuring these three base pairs with various relative positions in the $3 \times 3$ grid. For instance, the three base pairs shown in Figure 1 were also combined so that the horizontal pair was displayed in the same location as in Figure 1, but the other two pairs were moved, yielding a different multishape scene (Figure 2) in which base pairings were preserved.

This was done separately for the two sets. The three base pairs in each grid always comprised one pair grouped vertically, one pair grouped horizontally, and one pair grouped obliquely. Each base pair had four possible locations within the grid, and each pair always neighbored at least one 


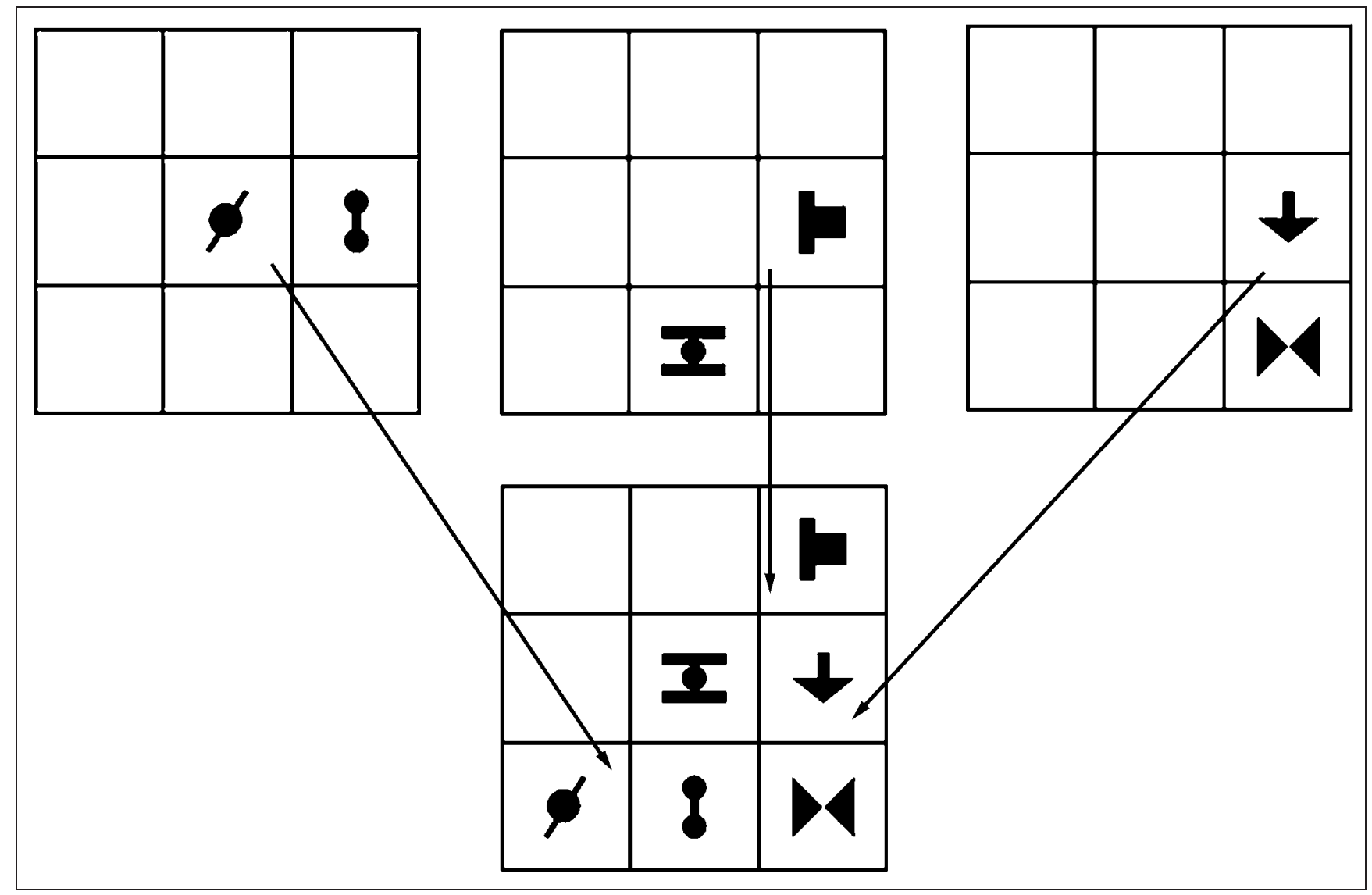

Figure 1. Construction of the grid array for the familiarization blocks from three base pairs.

other pair. This arrangement resulted in a total of 18 scenes constructed from the first set of pairs and 18 scenes constructed from the second set. Over all familiarization stimuli, the probability of appearance of any given element and of any given base pair was 0.5 . The probability of appearance of a non-base pair was 0.02. To guard against some shape pairings being more memorable than others, the assignment of shapes to shape pairs and to hemifields was randomized across participants.

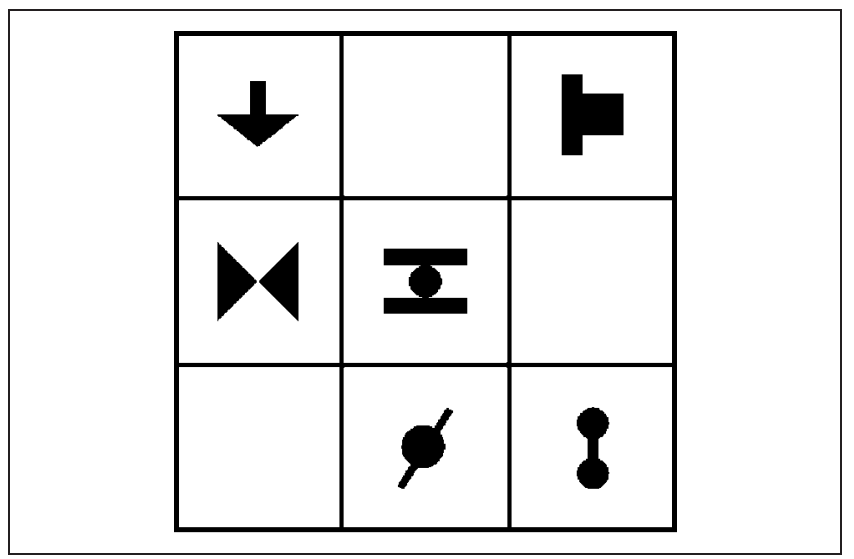

Figure 2. A second grid array for the familiarization blocks constructed by rearranging the three base pairs.
Participants were seated with their chin in a rest and their eyes approximately $57 \mathrm{~cm}$ from the screen. Eye gaze was tracked using an eye tracker with a remote infrared camera (SensoMotoric Instruments, Inc., Teltow, Germany). This system generated a transistor-to-transistor logic pulse within 30 msec (T. Dowe, personal communication, December 3, 2003) when a saccade was made at least $1.4^{\circ}$ into either the left or the right visual field. Stimuli were removed from the screen when this pulse was received by the computer controlling the experiment, thus preventing extended viewing. Deviations of eye position from the fixation point were recorded.

Before beginning the familiarization phase, participants were instructed to keep their gaze on the central fixation point. Task instructions were made intentionally vague to avoid informing participants as to the nature of the underlying structure. Participants were told simply to watch the series of stimuli to be presented to them and to take note of any regularities in the arrangement of the shapes. They were not informed of the spatial relationships between paired elements. These instructions, therefore, were appropriate for the investigation of learning in a relatively undirected context.

Participants were then presented with 10 runs of the 36 scenes in a random sequence. Gaze position was monitored by the experimenter in real time. Each trial was triggered by the experimenter when the subject's gaze was at 
fixation. Stimuli were displayed for approximately $3000 \mathrm{msec}$ after a delay of $1000 \mathrm{msec}$ after the trial was triggered. On each trial, the grid was displayed in only one visual field. The 18 scenes created using base pairs from Set 1 were presented to the left visual field (LVF), and the scenes created using Set 2 were presented to the right visual field (RVF). A short rest was taken after each block of 36 trials. After the familiarization phase participants were asked whether they had noticed any regularities in the arrays of shapes. They were then informed that certain shapes always appeared together in a particular arrangement and that different sets of shapes appeared on each side.

The test phase was run in four blocks immediately after familiarization and debriefing. In each block stimuli were displayed only on one side of the screen. Each trial was triggered by the experimenter when the subject's gaze was at fixation and stimuli were presented after a 1000-msec delay. On each trial, two grids (Figure 3) were presented sequentially for $3000 \mathrm{msec}$, separated by a 1000-msec delay during which the fixation point was displayed. Each grid contained only two shapes. On each trial, one grid contained a base pair from one of the sets and the other grid contained a non-base pair constructed from the shape elements that had been used to construct the base pairs within that set. The order of presentation of base and non-base pairs was randomized on each trial. There were six trials in each block with each base pair appearing twice. Participants were instructed to maintain fixation on the central point and to attend to the two pairs presented on each trial. Participants then had to make a two-alternative forced-choice (2AFC) and decide which pair of shapes had appeared together during the familiarization phase. Participants responded by pressing one of two buttons (Interval 1

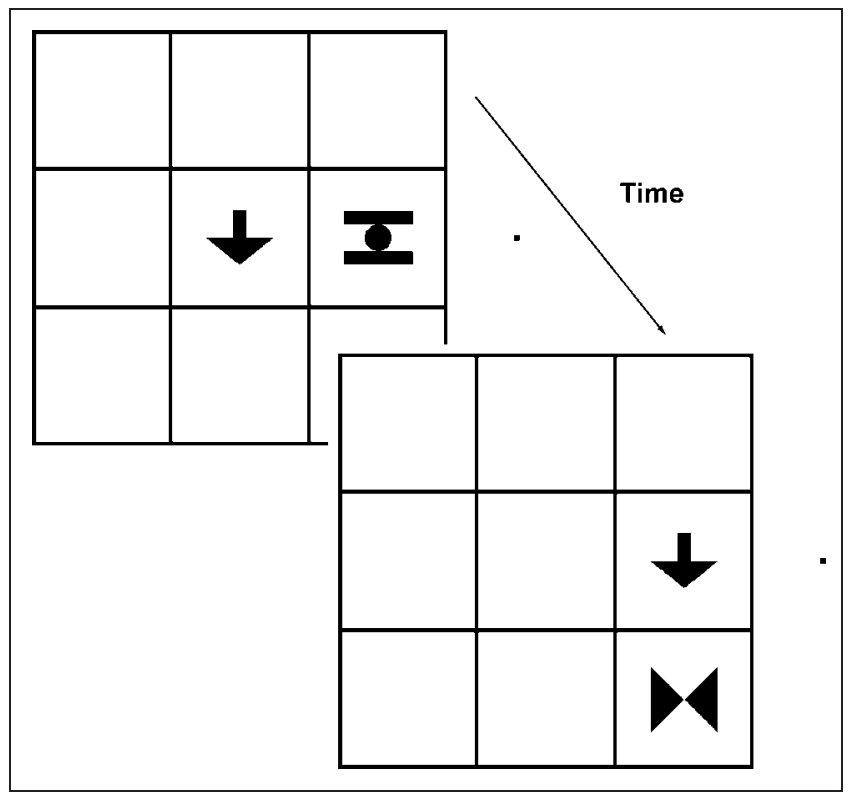

Figure 3. Sequential presentation of two grids containing a false and a true pair for the test blocks. Here grids are presented to the left of a fixation point. or Interval 2) with the hand on the same side as the visual field in which the stimuli were presented.

In the first two test blocks (LL = LVF familiarization/ LVF test; RR = RVF familiarization/RVF test), the base pairs and their accompanying non-base pairs were presented in the same visual field that they had appeared in during the familiarization phase. These will be referred to as ipsilateral tests. The order in which participants performed these two tests was randomized. In the third and fourth test blocks (contralateral tests), the base pairs and their accompanying non-base pairs were presented in the visual field opposite that in which they had appeared during the familiarization phase $(\mathrm{RL}=\mathrm{RVF}$ familiarization $/ \mathrm{LVF}$ test; $\mathrm{LR}=\mathrm{LVF}$ familiarization/RVF test). The order that participants performed these two tests was also randomized. Participants were informed to treat as irrelevant the side of the screen the shapes originally appeared on and the absolute positions of the shapes in the grid. They were instructed to only attend to the relative positions of the two shapes with respect to each other on the basis of their prior familiarization experience.

\section{Results}

Figure 4 shows the results from Experiment 1. Data from the group of young adult participants are labeled as the young group. Participants clearly chose the base pairs in preference to the random pairs in all test types (LL $=68.75 \%$, $S E M=6 \% ; \mathrm{RR}=71.88 \%, S E M=5 \% ; \mathrm{RL}=68.75 \%, S E M=$ $4.9 \%$; LR $=73.96 \%$, SEM $=5.7 \%$ ). There was a slight advantage in performance favoring the left hemisphere test conditions, but a $2 \times 2$ within-subject ANOVA with factors Practice Side (right-left) and Test Side (same-different) showed no main effect of either Practice Side, $F(1,15)=$ $.067, p>.79$, or Test Side, $F(1,15)=.065, p>.80$, and there was no sign of interaction between these factors, $F(1,15)=$ $1.27, p>.27$. In each of the four test conditions, the subjects' 2AFC performance was significantly different from chance performance (50\%) as measured by separate twotailed $t$ tests $(p<.005$ for each test). The overall level of performance (71.4\%) was not significantly different from the performance of participants who viewed the scenes foveally in Experiment 2 of Fiser and Aslin (2001) (62.1\%), $t(32)=1.56, p=.128$. Performance was compared with that of participants in Experiment 2 of Fiser and Aslin because positional learning could not contribute to abovechance performance. Although elements in non-base pairs did appear at test in locations where they had not appeared during familiarization, this was also true of elements in base pairs. Learning absolute element positions could not, therefore, be relied upon to distinguish between base pairs and non-base pairs in the current experiment. Similarly to Fiser and Aslin, none of the participants were able to notice or clearly describe the underlying regularity in the arrangement of the shapes, showing that they had no consistent conscious access to this information beyond their 2AFC judgments. This indicates that the minimally informative instructions to 


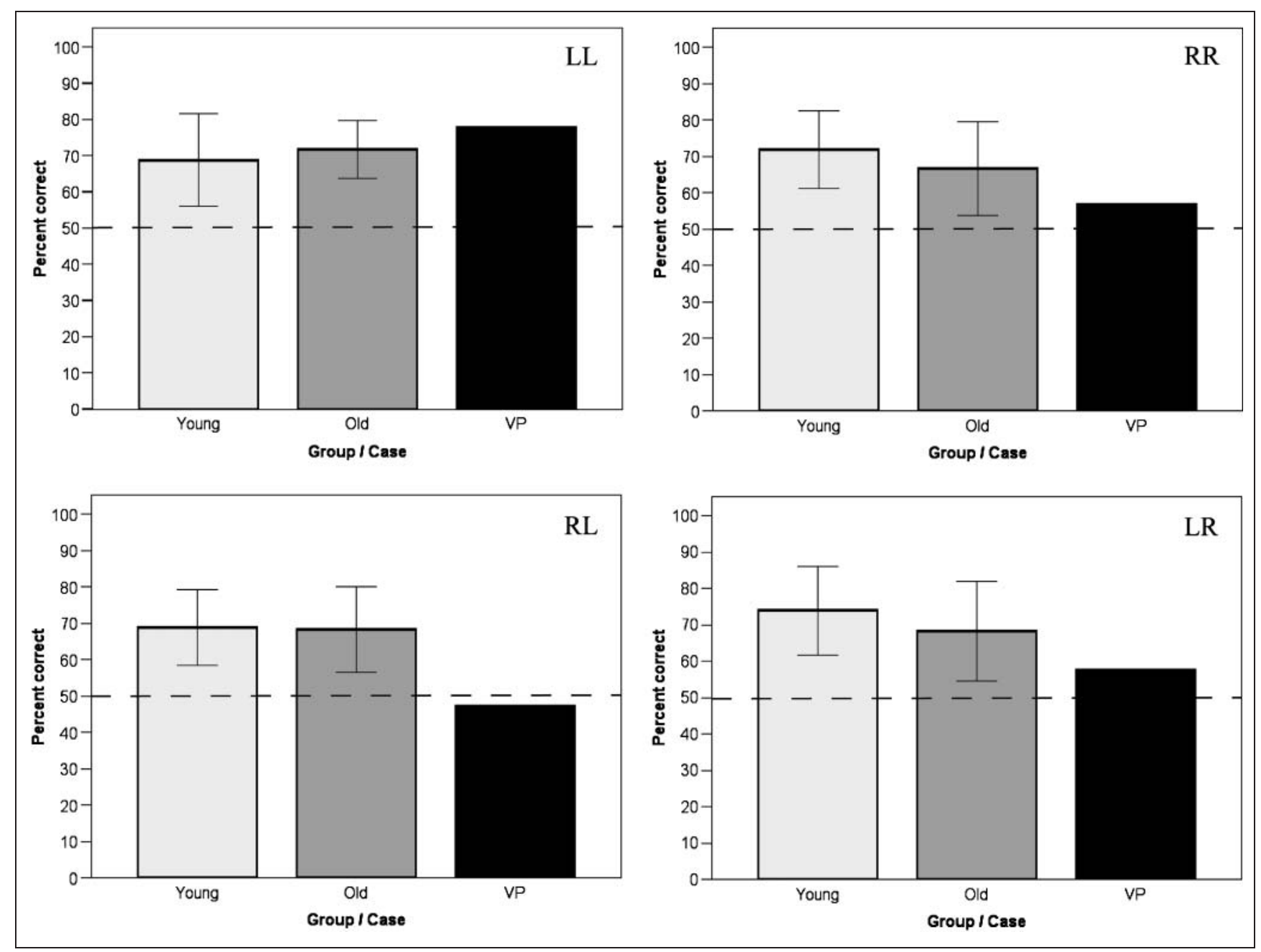

Figure 4. Percent correct for each of four test blocks for both groups of control participants and for patient V.P. Bars show $95 \%$ confidence intervals $(\mathrm{LL}=\mathrm{LVF}$ familiarization/LVF test; RR $=\mathrm{RVF}$ familiarization/RVF test; RL $=$ RVF familiarization/LVF test; LR $=\mathrm{LVF}$ familiarization/RVF test $)$.

take note of any regularities in arrangement did not lead to explicit awareness of the underlying base pair structure.

\section{EXPERIMENT 2}

Experiment 1 demonstrated that neurologically normal young adults can learn visual statistics with lateralized presentation of stimuli, establishing a baseline against which the performance of callosotomy patient V.P. could be measured. To rule out the possibility that any performance decrement exhibited by V.P. was due to a difference in age between V.P. and the younger control group, a second control group of 10 individuals aged matched to V.P. was tested. All participants were within 4 years of V.P.'s age at test and had normal or corrected-to-normal vision.

\section{Stimuli}

The stimuli were the same as those used in Experiment 1. Stimuli were displayed on a screen viewed from $66 \mathrm{~cm}$, and all elements subtended the same visual angles as the stimuli presented in Experiment 1.

\section{Procedure}

The procedure was identical to that used in Experiment 1, except for minor changes to the eye tracking procedure and the instructions given to participants. Eye gaze was tracked using an eye tracker with a remote infrared camera (SensoMotoric Instruments, Inc.). No transistor-to-transistor logic pulse communication between the eye tracker and stimulus computer was used. Gaze position was monitored in real time by the experimenter, and if gaze was seen to depart from this location, the participant was reminded to keep their eyes on the fixation point. No participants were reminded more than twice to maintain fixation.

Before the beginning of the familiarization phase, participants were given the exact instructions included in Appendix A. These instructions included no mention of regularities in the arrangement of stimuli. 


\section{Results}

Data from the group of age-matched adult participants are presented in Figure 4 and are labeled as the old group. The age-matched control group performed better than chance in all four test conditions ( $\mathrm{LL}=72 \%, S E M=4 \%$; $\mathrm{RR}=67 \%$, $S E M=6 \% ; \mathrm{RL}=68 \%, S E M=5 \% ; \mathrm{LR}=68 \%, S E M=6 \%)$. This was confirmed by two-tailed $t$ tests $(p<.05$ for each test) performed separately for each test condition. The overall level of performance (68.75\%) was not significantly different from the participants who viewed the scenes foveally in Experiment 2 of Fiser and Aslin (2001), $t(25)=$ $1.29, p=.208$, or from the younger participants in Experiment 1. As in Experiment 1, none of the participants in Experiment 2 were able to notice or clearly describe the underlying regularity in the arrangement of the shapes. Thus, the slight difference in task instructions between Experiments 1 and 2 was not accompanied by differences in awareness of the base pair structure. Instructing participants to attend to regularities did not lead to greater explicit awareness or increase 2AFC performance.

\section{EXPERIMENT 3}

Experiments 1 and 2 replicated the finding for statistical learning of visual regularities in neurologically normal adults found by Fiser and Aslin (2001) and extended this to stimuli presented outside of central vision and lateralized to the two visual hemifields. Experiment 3 investigated the effect of callosal disconnection on statistical learning.

\section{Methods}

\section{Participants}

One callosotomy patient, V.P., participated. At the time of testing, V.P. was a 52-year-old woman who underwent staged section of the corpus callosum in 1979 for the relief of intractable epilepsy. V.P. experienced recurrent seizures at 9 years of age. Anticonvulsant drugs controlled the seizures until 1979 when she began experiencing grand mal, petit mal, and myoclonic episodes while on multiple anticonvulsants. She underwent partial anterior callosal section in early April 1979, followed by complete section of her callosum in a second operation seven weeks later (Gazzaniga, Smylie, Baynes, Hirst, \& McCleary, 1984). V.P.'s detailed presurgical medical history is available elsewhere (Gazzaniga, Naas, Reeves, \& Roberts, 1984). Her postsurgical intelligence and memory quotients were within normal limits (Gazzaniga, Naas, et al., 1984). V.P. has been extensively tested in numerous lateralized paradigms over several years, and each isolated cerebral hemisphere has demonstrated good performance on many different tasks (for extensive reviews, see Gazzaniga, 2000, 2005), indicating that each can follow instructions and has intact perceptual (Corballis et al., 2002) and mnemonic (Holtzman \& Gazzaniga, 1985) abilities. As confirmed by MRI, the corpus callosum has been severed, except for a small cross section of fibers in the rostrum, in patient V.P. (Corballis, Inati, Funnell, Grafton, \& Gazzaniga, 2001). Figure 5 shows a midsagittal view of V.P.'s brain in which the absence of the corpus callosum, except for a small circled region, is apparent. Although the brains of callosotomy patients differ greatly from intact brains, evidence from both behavioral testing (Funnell, Corballis, \& Gazzaniga, 1999, 2003; Gazzaniga, Smylie, et al., 1984; Gazzaniga \& Smylie, 1983) and functional imaging studies (Hugdahl, 2000; Richter, Ugurbil, Georgopoulos, \& Kim, 1997; Cohen et al., 1996) suggests that the patterns of functional lateralization observed in the split brain are congruent with those found in neurologically intact participants in many different cognitive domains.

\section{Procedure}

Because of testing constraints, patient V.P. was tested over five consecutive days. Before familiarization, V.P. received the same instructions as the control participants in
Figure 5. Midsagittal MRIs of a normal brain with an intact corpus callosum and of patient V.P. showing clearly the complete absence of callosal fibers in V.P. except for a small region in the rostrum (circled). (MRI of V.P. provided by Souheil Inati, January 4, 2010.)

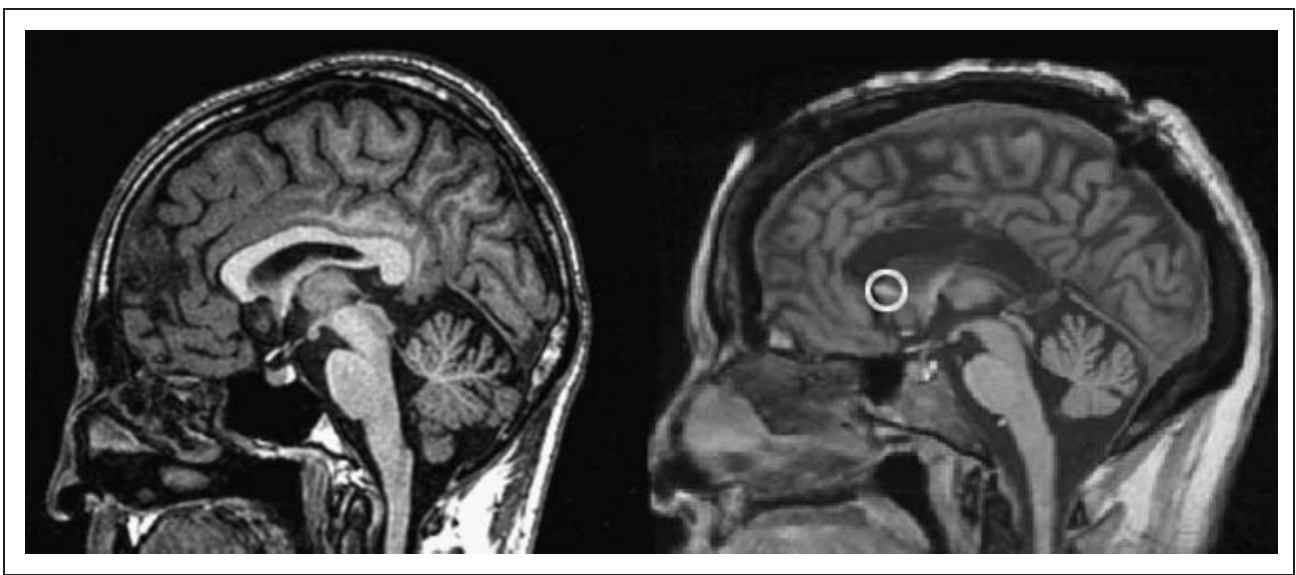


Experiment 1. On Day 1, V.P. completed six runs of the familiarization phase. On Day 2, a further four runs of familiarization were completed. V.P. was then informed that certain shapes always appeared together in a particular arrangement and that different sets of shapes appeared on each side. This explanation was accompanied by a display in free central vision of a visual aid consisting of a picture of the grid containing two letter stimuli in place of two shapes. This visual aid was therefore available to both cerebral hemispheres and was intended to compensate for any deficit in understanding linguistically presented instructions about spatial relationships between elements. No information about the actual relationships between shape elements was given.

V.P. then completed four runs of each of the ipsilateral tests, with the LVF tested first. She then completed four more familiarization runs followed by four blocks of each of the ipsilateral tests, with the RVF tested first. On Day 3, V.P. completed further familiarization runs followed by ipsilateral tests. On Days 4 and 5, V.P. completed several more familiarization runs and the contralateral tests. In total, V.P. completed 28 familiarization runs (vs. 10 runs in the control participants), 24 ipsilateral tests, and 12 contralateral tests (vs. 4 tests each in the control participants). This increased testing was undertaken to obtain as much data as possible from patient V.P. so as to maximize the accuracy of performance estimations and to maximize the potential for each hemisphere to demonstrate statistical learning. Repeated familiarization blocks were necessary to preserve the relationship between familiarization and test as in Experiment 1 in which testing followed immediately after a familiarization run. Notice that, until the beginning of tests on Day 4, V.P. saw half of the shapes only in one hemifield and the other half in the other hemifield during both the practice and the test trials. In both the patient and the control participants, the contralateral tests were carried out last. In both patient and controls, they followed familiarization blocks by only a few minutes.

The use of repeated testing, interspersed with familiarization blocks, raises the possibility of interference with statistical learning from non-base pairs. This possibility is remote given that testing was carried out on different days and always followed immediately after extended familiarization. Moreover, the number of presentations of non-base pair stimuli during test is miniscule compared with the number of presentations of non-base pair stimuli included within the familiarization displays. To address the concern that the presentation of base pairs in the initial tests may aid segmentation of base pairs from stimulus arrays in subsequent familiarization blocks, responses from only the first four ipsilateral test runs for each visual field were considered separately. These tests preceded repeated familiarization and are therefore immune from this concern. V.P. responded correctly on $83 \%$ of trials for the LVF and only $50 \%$ of trials for the RVF. This pattern of results for the data collected before repeated familiarization was essentially the same as the results for the entire data set, which are described below.

\section{Results}

Figure 4 shows the proportion of correct responses for each test type for patient V.P. displayed alongside the data for the control groups. Because of the single-subject design, the percentage of correct values for this individual were converted into measures of the detectability index, $d^{\prime}$, using tables provided in Hacker and Ratcliff (1979). Considering first the two ipsilateral tests, V.P. performed well above chance when stimuli were presented in the LVF $\left(d^{\prime}=1.09\right)$ and poorly when stimuli were presented in the RVF $\left(d^{\prime}=0.25\right)$. Performance in the two contralateral tests was poor; when familiarization stimuli were presented in the RVF and testing was carried out in the LVF, V.P. performed at chance $\left(d^{\prime}=-0.11\right)$. With familiarization in the left and testing in the RVFs, performance was close to chance $\left(d^{\prime}=0.29\right)$. When asked, before the commencement of the first test block, to describe the underlying regularity in the arrangement of the shapes, V.P. was unable to do so.

To further check for any potential effects of test order (LVF or RVF first after familiarization) or progressive learning over the two days of ipsilateral testing, percent correct was calculated separately for each testing session (Table 1). Right hemisphere (LVF) performance exceeded left hemisphere (RVF) performance and chance in every testing session and for both test orders. Although there is some suggestion that the left hemisphere

Table 1. Progression of Familiarization and Ipsilateral Test Blocks With Percent Correct

\begin{tabular}{lccc}
\hline Block & Test Order & Percent Correct LVF & Percent Correct RVF \\
\hline Familiarization Session 1 & $4 \times$ LVF then $4 \times$ RVF & 83 & 50 \\
Familiarization Session 2 & $4 \times$ RVF then $4 \times$ LVF & 67 & 58 \\
Familiarization Session 3 & $2 \times$ RVF then $2 \times \mathrm{LVF}$ & 83 & 58 \\
Familiarization Session 4 & $2 \times \mathrm{LVF}$ then $2 \times \mathrm{RVF}$ & 83 & 67 \\
Overall & & 78 & 57 \\
\hline
\end{tabular}


may have performed above chance in the final session, this calculated level of performance is based on fewer (12) responses than is the overall level of performance (72).

\section{DISCUSSION}

In the present study, the disconnection afforded by callosotomy opened a window into the asymmetry of cortical processing involved in visual statistical learning that is masked by hemispheric interaction in the normal brain. The results of Experiment 3 suggest that the learning of visual feature associations between elements in multishape scenes depends on the right cerebral hemisphere. V.P. only performed above chance when the co-occurrence statistics contained the subset of shapes that was both familiarized and tested in the LVF (right hemisphere). V.P.'s left hemisphere was unable to discriminate the base pairs from non-base pairs. Moreover, her poor performance in the two contralateral test blocks suggests that the corpus callosum is essential for the transfer of learned associations between the hemispheres.

These findings are consistent both with previous research showing that the isolated right hemisphere is able to perform some sophisticated perceptual functions that the left hemisphere cannot (Roser et al., 2005; Corballis et al., 1999) and with recent fMRI investigations of statistical learning reporting activations predominantly in righthemisphere regions at an early stage of familiarization with visually presented sequences of shapes (Turk-Browne et al., 2009). The results of the current study and the TurkBrowne et al. (2009) fMRI study are congruent with the hypothesis that visual statistical learning requires the involvement of right hemisphere mechanisms for the extraction of shape co-occurrence statistics. There are several reasons why this hypothesis is preferred over alternative explanations, invoking differences in procedures, age, or working memory capacity between the control group and the patient.

The deficit in performance, relative to control participants, shown by V.P.'s left hemisphere cannot be attributed to differences in the amount of familiarization. If anything, increased exposure to the stimuli should have increased learning. The slightly superior performance by V.P. than the control participants when both familiarized and tested in the LVF is probably the result of her much greater exposure to the learning materials. Nor can age differences between the patient and the control group account for the current finding. Although the neurologically intact participants in Experiment 1 were considerably younger than the patient, the effects of aging on hemispheric asymmetry and interaction have been found to be much smaller than the radical disconnection affected by severing the corpus callosum (Reuter-Lorenz \& Stanczak, 2000) and tend to be in the direction of the reduction of asymmetry (Cabeza, 2002). The ages of participants included in the Reuter-Lorenz and Stanczak (2000) study were also much older (65-
75 years) than the age of the callosotomy patient ( 52 years) in the present study. Thus, the striking dissociation of the performance of the two hemispheres is highly unlikely to be the result of age but is likely due to the surgical disconnection of the two hemispheres. Furthermore, Experiment 2 with control participants age-matched to the patient found evidence for statistical learning in both visual hemifields.

The 2AFC procedure used in the testing blocks involved maintaining visual information in working memory over the period of several seconds. The present results cannot be accounted for by hemispheric differences in working memory capacity as both hemispheres in the divided brain, including in patient V.P., have demonstrated the ability to maintain information in such a fashion (Holtzman \& Gazzaniga, 1982, 1985). Studies with split-brain monkeys have also shown that both isolated hemispheres are able to maintain a representation of serially presented stimuli (Lewine, Doty, Astur, \& Provencal, 1994).

If our assessment about the right hemisphere being crucially involved in visual statistical learning is correct, then the present results also shed an interesting light on the general process of how visual learning occurs in the brain. Recent investigations using complex visual pictures suggest that scenes are abstracted to the categorical level automatically and that statistical learning of categorical associations can proceed incidentally (Brady \& Oliva, 2008). Categorical-level learning may proceed in tandem with low-level learning, as identification of associated items was found to be strongest when both categorical and perceptual regularities were present in a stream of visual images. This raises the possibility that statistical learning at these two levels of abstraction may be mediated by, at least partially, separate systems in the brain each differentially engaging cortical areas typically involved in perceptual and categorical processing. Our results show that statistical learning of visuospatial associations between objects can proceed in the isolated right hemisphere of the brain that has previously demonstrated an inability to draw simple inferences (Roser et al., 2005), suggesting a low-level, perceptual process. Whether categorical statistical learning is similarly lateralized remains to be determined.

Our results may also generate hypotheses about possible patterns of brain activity as statistical learning progresses from the construction of perceptual associations to the formation of abstract and explicit conceptual knowledge. Learning visual statistics without explicit awareness has been found to involve activity in right hemisphere regions also involved in implicit learning, at least in the early phase of familiarization (Turk-Browne et al., 2009). It has also been demonstrated recently that visual statistical learning results in the creation of representations of object associations that are sufficiently abstract to allow for the transfer of learning across and between temporal and spatial dimensions, despite not being accompanied by explicit knowledge of visual statistics (Turk-Browne \& Scholl, 2009). 
Developing explicit representations of visual regularities requires extended exposure (Seger et al., 2000). This transition from low- to high-level representation may be reflected in a change in brain processes.

According to the accepted view, in the normal brain the products of a lateralized perceptual process may eventually be made available to higher order cognitive processes. The generation, testing, and application of explicit hypotheses about incoming information has been associated with the left hemisphere through the testing of split-brain patients (Gazzaniga, 2000) and by neuroimaging studies of reasoning (Noveck, Goel, \& Smith, 2004; Goel \& Dolan, 2003). In a neuroimaging study of visual-concept learning in a classification task, Seger et al. (2000) found that the initial phase of learning activated right parietal and frontal regions. As learning progressed activation in left parietal cortex increased and left prefrontal activation was observed only in those participants who developed explicit conceptual knowledge of the stimulus material. Similarly, the formation of explicit conceptual knowledge of stimulus associations in observational learning may depend on the integration of the products of a right lateralized perceptual process with systems supporting conceptual knowledge by the left hemisphere. This must depend on interhemispheric transfer via the corpus callosum as the disruption of this commissure has been shown to eliminate the transfer of all but the most rudimentary of information between the hemispheres (Corballis, 1994), and the corpus callosum has been shown to be crucial for the transfer of learning between the hemispheres (Sperry, Stamm, \& Miner, 1956). As described in the Introduction, this view leaves open, whether in the case of abolished transfer between the two, the right or the left hemisphere can handle visual learning alone.

Investigation of visual statistical learning in the split brain allowed the hemispheres to be tested in relative isolation and allowed the dissociation of visual statistical learning and explicit conceptual knowledge. Our results suggest that explicit conceptual top-down effects from the left hemisphere were not required for successful visual learning in the right hemisphere, as the left hemisphere could not learn the patterns even when V.P. was given an explicit conceptual description of what she needed to look for. Conversely, visual statistical learning was successful in the right hemisphere in patient V.P. that has been shown to represent information in a veridical, noninterpretive manner (Gazzaniga, 2000) and to be unable to make simple inferences about causes from associative information at the two-year-old level (Roser et al., 2005). This combination of a right-hemisphere deficit in drawing explicit inference and right-hemisphere competence in visual statistical learning suggests that the learning of visual feature associations between elements in multishape scenes depends on implicit processes. These aforementioned tests (Roser et al., 2005) were conducted with explicit linguistic and visual instructions. The task we used to assess visual statisti- cal learning does not require explicit knowledge (although it may be present in some participants) but does necessitate that the learner develop an abstract implicit representation of the visual input. We also showed that these results can be captured very well by a model that assumes implicit hypothesis testing with each visual scene, by which the most likely underlying structure of the scene is inferred (Orban et al., 2008). Therefore, our results are consistent with the view that statistical learning is not only incidental and automatic but also a product of an implicit generation of hypotheses that can be handled in the right hemisphere alone.

This view is also congruent with several models of functional laterality (Dien, 2008). For instance, it has been proposed that the right hemisphere handles novel stimuli and situations whereas the left hemisphere dominates behavior when extensive experience has allowed the formation of internal routines for describing and reacting to the external environment (Goldberg \& Costa, 1981). In a similar vein, a general processing asymmetry on the basis of spatial frequency and the hierarchy (global/local) of elements within a visual scene (Grabowska \& Nowicka, 1996; Sergent, 1982) has been proposed. The right hemisphere has been identified with global processing of visual information by a variety of methods, including the testing of callosotomy and unilateral-lesion patients, electrophysiology, and functional imaging (Dien, 2008). The learning of image statistics through observation, such as in the present study, involves the generation of higher order, or global, representations of scenes from smaller constituent items, explaining the advantage of the right hemisphere. These various models of functional asymmetry recognize that cortical lateralization tends to be relative rather than absolute and subject to influence by numerous factors, such as the nature of the task (Banich, 1998) and the stimulus material (Kelley et al., 1998). Thus, our finding of a righthemisphere role in learning associations between visual shapes in set spatial arrangements may lead to two conclusions: Either the right hemisphere is highly involved in the initial learning of associations per se or the asymmetry in performance is due to the visual-spatial nature of the stimuli. As the learning of artificial grammar has been found to involve increasing left fronto-parietal functional connectivity (Fletcher, Buchel, Josephs, Friston, \& Dolan, 1999), it is plausible that a left hemisphere advantage for learning associations between linguistic stimuli (words or syllables) may be observed in future research. The current finding adds to the large body of evidence from neurological case studies describing asymmetry of cortical function and, importantly, demonstrates visual statistical learning in the absence of higher conceptual knowledge, thus informing as to the nature of this fundamental process. Moreover, functional imaging of visual statistical learning in neurologically normal participants may reveal an initial reliance on right-hemispheric networks, followed by a transition to more bilateral activity as learning progresses to full conceptual knowledge. 


\section{APPENDIX A}

\section{Instructions to Age-matched Control Participants}

Your task is to simply view some grids with shapes arranged in them whilst keeping your gaze on the central fixation point. Pay attention to and take note of what is presented in the grids. We will run 10 blocks, each of 36 trials. There will be a short break between each block. Your gaze will be monitored in real time. Please do not look directly at the stimuli. The experimenter will remind you if they see your gaze wandering.

\section{Acknowledgments}

This research was supported by the National Institutes of Health grant no. RO1 MH059825 to M. S. Gazzaniga.

Reprint requests should be sent to Matthew E. Roser, School of Psychology University of Plymouth, Plymouth PL4 8AA, Devon, United Kingdom, or via e-mail: matt.roser@plymouth.ac.uk.

\section{REFERENCES}

Baker, C. I., Olson, C. R., \& Behrmann, M. (2004). Role of attention and perceptual grouping in visual statistical learning. Psychological Science, 15, 460-466.

Banich, M. T. (1998). The missing link: The role of interhemispheric interaction in attentional processing. Brain and Cognition, 36, 128-157.

Baynes, K., Eliassen, J. C., Lutsep, H. L., \& Gazzaniga, M. S. (1998). Modular organization of cognitive systems masked by interhemispheric integration. Science, 280, 902-905.

Biederman, I. (1987). Recognition-by-components-A theory of human image understanding. Psychological Review, 94, 115-147.

Bogen, J. E., \& Gazzaniga, M. S. (1965). Cerebral commissurotomy in man. Minor hemisphere dominance for certain visuospatial functions. Journal of Neurosurgery, 23, 394-399.

Brady, T. F., \& Oliva, A. (2008). Statistical learning using real-world scenes: Extracting categorical regularities without conscious intent. Psychological Science, 19, 678-685.

Cabeza, R. (2002). Hemispheric asymmetry reduction in older adults: The HAROLD model. Psychology and Aging, 17, 85-100.

Cohen, J. D., MacWhinney, B., Flatt, M., \& Provost, J. (1993). PsyScope: A new graphic interactive environment for designing psychology experiments. Behavior Research Methods, Instruments, \& Computers, 25, 257-271.

Cohen, M. S., Kosslyn, S. M., Breiter, H. C., DiGirolamo, G. J., Thompson, W. L., Anderson, A. K., et al. (1996). Changes in cortical activity during mental rotation: A mapping study using functional MRI. Brain, 119, 89-100.

Conway, C. M., \& Christiansen, M. H. (2005). Modality-constrained statistical learning of tactile, visual, and auditory sequences. Journal of Experimental Psychology: Learning Memory and Cognition, 31, 24-39.

Corballis, M. C. (1994). Split decisions: Problems in the interpretation of results from commissurotomized subjects. Behavioural Brain Research, 64, 163-172.

Corballis, M. C., \& Sergent, J. (1988). Imagery in a commissurotomized patient. Neuropsychologia, 26, 13-26.

Corballis, P. M. (2003). Visuospatial processing and the right-hemisphere interpreter. Brain and Cognition, 53, $171-176$
Corballis, P. M., Fendrich, R., Shapley, R. M., \& Gazzaniga, M. S. (1999). Illusory contour perception and amodal boundary completion: Evidence of a dissociation following callosotomy. Journal of Cognitive Neuroscience, 11, 459-466.

Corballis, P. M., Funnell, M. G., \& Gazzaniga, M. S. (2002). Hemispheric asymmetries for simple visual judgments in the split brain. Neuropsychologia, 40, 401-410.

Corballis, P. M., Inati, S., Funnell, M. G., Grafton, S. T., \& Gazzaniga, M. S. (2001). MRI assessment of spared fibers following callosotomy: A second look. Neurology, 57, $1345-1346$.

Creel, S. C., Newport, E. L., \& Aslin, R. N. (2004). Distant melodies: Statistical learning of nonadjacent dependencies in tone sequences. Journal of Experimental Psychology: Learning Memory and Cognition, 30, 1119-1130.

Dien, J. (2008). Looking both ways through time: The Janus model of lateralized cognition. Brain and Cognition, 67, 292-323.

Fiser, J., \& Aslin, R. N. (2001). Unsupervised statistical learning of higher-order spatial structures from visual scenes. Psychological Science, 12, 499-504.

Fiser, J., \& Aslin, R. N. (2002a). Statistical learning of higher-order temporal structure from visual shape sequences. Journal of Experimental Psychology: Learning Memory and Cognition, 28, 458-467.

Fiser, J., \& Aslin, R. N. (2002b). Statistical learning of new visual feature combinations by infants. Proceedings of the National Academy of Sciences, U.S.A., 99, 15822-15826.

Fiser, J., \& Aslin, R. N. (2005). Encoding multielement scenes: Statistical learning of visual feature hierarchies. Journal of Experimental Psychology: General, 134, 521-537.

Fiser, J., Scholl, B. J., \& Aslin, R. N. (2007). Perceived object trajectories during occlusion constrain visual statistical learning. Psychonomic Bulletin \& Review, 14, 173-178.

Fletcher, P., Buchel, C., Josephs, O., Friston, K., \& Dolan, R. (1999). Learning-related neuronal responses in prefrontal cortex studied with functional neuroimaging. Cerebral Cortex, 9, 168-178.

Fugelsang, J. A., Roser, M. E., Corballis, P. M., Gazzaniga, M. S., \& Dunbar, K. N. (2005). Brain mechanisms underlying perceptual causality. Cognitive Brain Research, 24, 41-47.

Funnell, M. G., Corballis, P. M., \& Gazzaniga, M. S. (1999). A deficit in perceptual matching in the left hemisphere of a callosotomy patient. Neuropsychologia, 37, 1143-1154.

Funnell, M. G., Corballis, P. M., \& Gazzaniga, M. S. (2003). Temporal discrimination in the split brain. Brain and Cognition, 53, 218-222.

Gazzaniga, M. S. (2000). Cerebral specialization and interhemispheric communication: Does the corpus callosum enable the human condition? Brain, 123, 1293-1326.

Gazzaniga, M. S. (2005). Forty-five years of split-brain research and still going strong. Nature Reviews Neuroscience, 6, 653-659.

Gazzaniga, M. S., Naas, R., Reeves, A., \& Roberts, D. (1984). Neurologic perspectives on right hemisphere language following surgical section of the corpus callosum. Seminars in Neurology, 4, 126-135.

Gazzaniga, M. S., \& Smylie, C. S. (1983). Facial recognition and brain asymmetries-Clues to underlying mechanisms. Annals of Neurology, 13, 536-540.

Gazzaniga, M. S., Smylie, C. S., Baynes, K., Hirst, W., \& McCleary, C. (1984). Profiles of right-hemisphere language and speech following brain bisection. Brain and Language, 22, 206-220.

Goel, V., \& Dolan, R. J. (2003). Explaining modulation of reasoning by belief. Cognition, 87, B11-B22.

Goldberg, E., \& Costa, L. D. (1981). Hemisphere differences in the acquisition and use of descriptive systems. Brain and Language, 14, 144-173. 
Grabowska, A., \& Nowicka, A. (1996). Visual-spatial-frequency model of cerebral asymmetry: A critical survey of behavioral and electrophysiological studies. Psychological Bulletin, 120, 434-449.

Hacker, M. J., \& Ratcliff, R. (1979). A revised table of $d^{\prime}$ for m-alternative forced choice. Perception \& Psychophysics, 26, 168-170.

Hauser, M. D., Newport, E. L., \& Aslin, R. N. (2001). Segmentation of the speech stream in a non-human primate: Statistical learning in cotton-top tamarins. Cognition, 78, B53-B64.

Holtzman, J. D., \& Gazzaniga, M. S. (1982). Dual task interactions due exclusively to limits in processing resources. Science, 218, 1325-1327.

Holtzman, J. D., \& Gazzaniga, M. S. (1985). Enhanced dual task performance following corpus commissurotomy in humans. Neuropsychologia, 23, 315-321.

Hugdahl, K. (2000). Lateralization of cognitive processes in the brain. Acta Psychologica, 105, 211-235.

Kelley, W. M., Miezin, F. M., McDermott, K. B., Buckner, R. L., Raichle, M. E., Cohen, N. J., et al. (1998). Hemispheric specialization in human dorsal frontal cortex and medial temporal lobe for verbal and nonverbal memory encoding. Neuron, 20, 927-936.

Kirkham, N. Z., Slemmer, J. A., \& Johnson, S. P. (2002). Visual statistical learning in infancy: Evidence for a domain general learning mechanism. Cognition, 83, B35-B42.

Lewine, J. D., Doty, R. W., Astur, R. S., \& Provencal, S. L. (1994). Role of the forebrain commissures in bihemispheric mnemonic integration in macaques. Journal of Neuroscience, 14, 2515-2530.

MacKenzie, K., \& Fiser, J. (2008). Sensitivity of implicit visual rule-learning to the saliency of the stimuli. Journal of Vision, 8, 474.

Marr, D. (1982). Vision: A computational investigation into the buman representation and processing of visual information. San Francisco: W. H. Freeman.

Noveck, I. A., Goel, V., \& Smith, K. W. (2004). The neural basis of conditional reasoning with arbitrary content. Cortex, 40, 613-622.

Orban, G., Fiser, J., Aslin, R. N., \& Lengyel, M. (2008). Bayesian learning of visual chunks by human observers. Proceedings of the National Academy of Sciences, U.S.A., 105, 2745-2750.

Perruchet, P., \& Pacton, S. (2006). Implicit learning and statistical learning: One phenomenon, two approaches. Trends in Cognitive Sciences, 10, 233-238.
Phelps, E. A., \& Gazzaniga, M. S. (1992). Hemispheric differences in mnemonic processing: The effects of left hemisphere interpretation. Neuropsychologia, 30, 293-297.

Reuter-Lorenz, P. A., \& Stanczak, L. (2000). Differential effects of aging on the functions of the corpus callosum. Developmental Neuropsychology, 18, 113-137.

Richter, W., Ugurbil, K., Georgopoulos, A., \& Kim, S. G. (1997). Time-resolved fMRI of mental rotation. NeuroReport, 8 , 3697-3702.

Roser, M. E., Fugelsang, J. A., Dunbar, K. N., Corballis, P. M., \& Gazzaniga, M. S. (2005). Dissociating processes supporting causal perception and causal inference in the brain. Neuropsychology, 19, 591-602.

Saffran, J. R., Aslin, R. N., \& Newport, E. L. (1996). Statistical learning by 8-month-old infants. Science, 274, 1926-1928.

Saffran, J. R., Newport, E. L., \& Aslin, R. N. (1996). Word segmentation: The role of distributional cues. Journal of Memory and Language, 35, 606-621.

Saffran, J. R., Pollak, S. D., Seibel, R. L., \& Shkolnik, A. (2007). Dog is a dog is a dog: Infant rule learning is not specific to language. Cognition, 105, 669-680.

Seger, C. A., Poldrack, R. A., Prabhakaran, V., Zhao, M., Glover, G. H., \& Gabrieli, J. D. E. (2000). Hemispheric asymmetries and individual differences in visual concept learning as measured by functional MRI. Neuropsychologia, 38, 1316-1324.

Sergent, J. (1982). The cerebral balance of power: Confrontation or cooperation? Journal of Experimental Psychology: Human Perception and Performance, 8, 253-272.

Sperry, R. W., Stamm, J. S., \& Miner, N. (1956). Relearning tests for interocular transfer following division of optic chiasma and corpus callosum in cats. Journal of Comparative $\mathcal{E}$ Physiological Psychology, 49, 529-533.

Turk-Browne, N. B., Junge, J. A., \& Scholl, B. J. (2005). The automaticity of visual statistical learning. Journal of Experimental Psychology: General, 134, 552-564.

Turk-Browne, N. B., \& Scholl, B. J. (2009). Flexible visual statistical learning: Transfer across space and time. Journal of Experimental Psychology: Human Perception and Performance, 35, 195-202.

Turk-Browne, N. B., Scholl, B. J., Chun, M. M., \& Johnson, M. K. (2009). Neural evidence of statistical learning: Efficient detection of visual regularities without awareness. Journal of Cognitive Neuroscience, 21, 1934-1945.

Wolford, G., Miller, M. B., \& Gazzaniga, M. S. (2000). The left hemisphere's role in hypothesis formation. Journal of Neuroscience, 20, RC64. 